ISSN 0103-9954

\title{
PARTIÇÃO ESPACIAL DE ESPÉCIES ARBÓREAS EM FUNÇÃO DA DRENAGEM DO SOLO EM UM FRAGMENTO DE FLORESTA COM ARAUCÁRIA NO SUL DO BRASIL
}

\section{TREE SPECIES COMMUNITY SPATIAL PARTITION IN FUNCTION OF SOIL DRAINAGE IN AN ARAUCARIA FOREST FRAGMENT IN SOUTHERN BRAZIL}

\author{
Pedro Higuchi ${ }^{1}$ Ana Carolina da Silva ${ }^{2}$ Manoela Drews de Aguiar ${ }^{3}$ Álvaro Luiz Mafra ${ }^{4}$ \\ Marcelo Negrini ${ }^{5}$ Diego Fernando Zech ${ }^{6}$
}

\section{RESUMO}

O estudo das relações entre a vegetação e os solos pode auxiliar na compreensão da estrutura de uma comunidade florestal, dando suporte à conservação da biodiversidade. Assim, o presente estudo teve como objetivo avaliar a existência da partição espacial da comunidade de espécies arbóreas em um fragmento de Floresta com Araucária, em função da drenagem do solo. Para este propósito, foi realizada a caracterização ambiental (drenagem do solo, propriedades físicas e químicas do solo, topografia, compactação dos solos, profundidade dos solos e cobertura do dossel) em 25 parcelas de $20 \times 20 \mathrm{~m}$, onde indivíduos de espécies arbóreas, com circunferência a altura do peito (CAP) maior ou igual a $15,7 \mathrm{~cm}$, foram previamente contados, medidos e identificados. Os dados foram analisados por meio do teste de Mann-Withney, análise de variância multivariada não paramétrica (NPMANOVA), análise multivariada (NMDS) e análise de espécies indicadoras. Os locais foram classificados como bem e moderadamente drenados, com diferenças ambientais que determinaram a riqueza, a partição espacial da comunidade e a ocorrência de espécies indicadoras na pequena escala espacial considerada. Desta forma, conclui-se que, no fragmento florestal estudado, as variações espaciais na drenagem do solo foram determinantes na heterogeneidade da composição florísticaestrutural observada.

Palavras-chave: biodiversidade; Mata Atlântica; umidade do solo.

\section{ABSTRACT}

The relationship vegetation-soil can contribute to understand the forest structure, supporting biodiversity conservation. Thus, the aim of the present study was to verify the existence of spatial partition of the tree species community in an Araucaria forest fragment in function of soil drainage. For this sake, an environmental characterization (soil drainage, physical and chemical soil properties, topography, compression

1 Engenheiro Florestal, Dr., Professor do Departamento de Engenharia Florestal, Centro de Ciências Agroveterinárias, Universidade do Estado de Santa Catarina, Av Luiz de Camões, 2090 - Conta Dinheiro, CEP 88520-000, Lages (SC), Brasil. higuchip@gmail.com

2 Engenheira Florestal, Dr., Professora, Departamento de Engenharia Florestal, Centro de Ciências Agroveterinárias, Universidade do Estado de Santa Catarina, Av Luiz de Camões, 2090 - Conta Dinheiro, CEP 88520-000, Lages (SC), Brasil. carol_sil4@yahoo.com.br

3 Engenheira Florestal, Departamento de Engenharia Florestal, Centro de Ciências Agroveterinárias, Universidade do Estado de Santa Catarina, Av Luiz de Camões, 2090 - Conta Dinheiro, CEP 88520-000, Lages (SC), Brasil. manoo_aguiar@hotmail.com

4 Engenheiro Agrônomo, Dr., Departamento de Solos, Centro de Ciências Agroveterinárias, Universidade do Estado de Santa Catarina, Av Luiz de Camões, 2090 - Conta Dinheiro, CEP 88520-000, Lages (SC), Brasil. a2alm@cav.udesc.br

5 Engenheiro Florestal, Departamento de Engenharia Florestal, Centro de Ciências Agroveterinárias, Universidade do Estado de Santa Catarina, Av Luiz de Camões, 2090 - Conta Dinheiro, CEP 88520-000, Lages (SC), Brasil. engfmarcelonegrini@hotmail.com

6 Engenheiro Florestal, Departamento de Engenharia Florestal, Centro de Ciências Agroveterinárias, Universidade do Estado de Santa Catarina, Av Luiz de Camões, 2090 - Conta Dinheiro, CEP 88520-000, Lages (SC), Brasil. diegozech@gmail.com 
with circumference at breast height $\geq 15.7 \mathrm{~cm}$ were previously counted, measured and identified. The data were analysed by Mann-Withney test, non-parametric multivariate ANOVA (NPMANOVA), multivariate analysis (NMDS) and indicator species analysis. In this small spatial scale there were two drainage classes, corresponding to well and moderately-drained soils, with environmental differences that determined the richness, the spatial partition of the tree community and the occurrence of indicator species. Thus, we conclude that in the study forest fragment soil drainage spatial variations were determinant in the floristicstructural heterogeneity observed in tree community.

Keywords: Atlantic Forest; biodiversity; soil moisture.

of soil, depth of soil and canopy cover) was realized in 25 plots of $20 \times 20 \mathrm{~m}$, where tree individuals, INTRODUÇÃO

Dentre as diferentes formações florestais que compõem a Floresta Atlântica brasileira, merece destaque a Floresta com Araucária, classificada como Floresta Ombrófila Mista (IBGE, 1992), e caracterizada pela distribuição atual restrita em áreas de elevada altitude nas regiões sul e sudeste do Brasil. Nestes locais, há predomínio de baixas temperaturas, que conferem fisionomia e composição florística peculiares. $\mathrm{O}$ aspecto de homogeneidade, devido ao domínio da Araucaria angustifolia (Bertol.) Kuntze no dossel, esconde a elevada variação florística que ocorre em função de variações no clima e de gradientes altitudinais (HIGUCHI et al., 2012a). Sua importância se estende às esferas econômica e social, devido à existência de várias espécies de interesse madeireiro e não madeireiro (e.g. Arancaria angustifiolia, Mimosa scabrella Benth., Ocotea spp., Ilex paraguariensis A.St.-Hil.).

Em função do histórico processo de ocupação de terras, caracterizado pelo corte seletivo e expansão de áreas urbanas e de atividades agropecuárias e, mais recentemente, silviculturais, esta floresta atualmente se encontra fragmentada (RIBEIRO et al., 2009), com remanescentes em diferentes níveis de degradação. Este cenário demonstra a necessidade de estudos que tenham como meta subsidiar estratégias de conservação e manejo, com intuito de uso sustentável dos recursos florestais e restauração ecológica de áreas degradadas. Neste sentido, o entendimento da organização espacial de comunidades de espécies arbóreas em fragmentos florestais assume grande relevância para a definição dessas estratégias.

Estudos têm demonstrado que, em fragmentos de Floresta com Araucária, parte da organização da comunidade de espécies arbóreas é explicada pela heterogeneidade ambiental existente (e.g. BARDDAL et al., 2004; CARVALHO et al., 2009; HIGUCHI et al., 2012b). Esta relação, espécies e ambiente, é resultado de uma longa história evolutiva, onde eventos relacionados à fitogeografia, como, por exemplo, a expansão e a retração da vegetação em reposta a paleoeventos climáticos, determinam um "pool" regional de espécies que, a partir de forças relacionadas à competição e filtros ambientais, determinam uma comunidade local, bem como suas variações em pequenas escalas espaciais (WEBB et al., 2002).

Em se tratando de filtro ambiental, a baixa capacidade de drenagem do solo é considerada um importante fator seletivo, sendo que a topografia, a estrutura e o arranjo dos poros no solo determinam o fornecimento de água para as plantas e a capacidade de infiltração no perfil (SOLLINS, 1998) e, consequentemente, sua saturação hídrica ou não. No caso de acúmulo de água, a aeração do soloé reduzida, com forte influência sobre o desenvolvimento de espécies vegetais (SOLLINS, 1998). Em função de condições de baixa disponibilidade de oxigênio, várias espécies arbóreas apresentam adaptações que permitem o desenvolvimento destas em áreas sujeitas ao alagamento, como as de origem metabólicas, morfológicas e anatômicas (PIMENTA et al., 1998; SILVA et al., 2012a). Assim, é natural que vários estudos (OLIVEIRA FILHO et al., 1997; SOLLINS, 1998; SILVA et al., 2007; BUDKE et al., 2008; SILVA et al., 2009; SILVA et al., 2010; STEEGE et al., 1993) relatem que drenagem do solo e diferentes regimes de inundação representem fator determinante na distribuição das espécies arbóreas em ecossistemas florestais. No entanto, no que se refere à Floresta com Araucária, pouco se conhece sobre a organização de comunidades arbóreas em função de diferentes classes de drenagem do solo.

Neste contexto, o papel das formações florestais em relação aos solos e recursos hídricos é de especial interesse, sobretudo em diversas 
regiões do planalto sul brasileiro, que corresponde às cabeceiras de importantes rios da bacia do Uruguai, além de coincidirem com zonas de recarga do Aquífero Guarani (GOMES et al., 2008). Desta forma, com o propósito de contribuir para o conhecimento da ecologia de fragmentos florestais na região Sul do Brasil, o presente estudo teve como objetivo verificar a existência de partição espacial de uma comunidade de espécies arbóreas em um fragmento de Floresta com Araucária no Estado de Santa Catarina em função da drenagem do solo.

\section{MATERIAL E MÉTODO}

\section{Área de Estudo}

$\mathrm{O}$ estudo foi realizado em um remanescente de Floresta com Araucária (2751'04"S; $50^{\circ} 19^{\prime} 07^{\prime}$ 'W, altitude $980 \mathrm{~m}$ ), com área total de 22 ha, localizado no município de Lages, no Planalto Catarinense (Figura 1). O clima é mesotérmico úmido com verões amenos, $\mathrm{Cfb}$ de acordo com a classificação de Köppen. A precipitação média anual é de $1.479 \mathrm{~mm}$, bem distribuída ao longo do ano, com temperatura média anual de $16^{\circ} \mathrm{C}$. O fragmento florestal ocorre em uma microbacia hidrográfica com predomínio de relevo ondulado. Os solos da área de estudo foram desenvolvidos na zona de contato de rochas efusivas da Formação Serra Geral, com sedimentos Jurocretáceos e Permianos, das Formações Botucatu e Rio do Rastro, respectivamente (EMBRAPA, 2004).

\section{Desenho amostral e coleta de dados}

A coleta de dados foi realizada em
25 parcelas permanentes de $20 \times 20 \mathrm{~m}$, totalizando 1 ha de área amostrada (Figura 1), alocadas em 2008 para a caracterização do componente arbóreo realizado por Silva et al. (2012b). Neste estudo, todos os indivíduos arbóreos vivos com circunferência a altura do peito (CAP) igual ou superior a $15,7 \mathrm{~cm}$ foram medidos (CAP), identificados e classificados de acordo com a APG III (CHASE et al., 2009). As parcelas foram distribuídas no fragmento de forma sistemática estratificada, de forma que, em cada estrato, as parcelas situaram-se $20 \mathrm{~m}$ entre si. A distribuição dos estratos foi definida com o propósito de contemplar adequadamente a variação ambiental existente, tais como borda/interior, variações topográficas e solos com drenagens distintas.

As classes de drenagem e de solo em cada parcela foram definidas de acordo com a metodologia proposta por, respectivamente, Santos et al. (2005) e EMBRAPA (2006). As variáveis topográficas de cada parcela foram representadas pela cota média (m) dos quatro vértices, pelo desnível máximo, calculado a partir da diferença do valor de cota entre os vértices de maior e menor altitude, e pela declividade média, definida pela declividade média dos quatro lados de cada parcela (OLIVEIRA FILHO et al., 1994). As variáveis químicas e físicas do solo foram avaliadas em amostras compostas de 10 subamostras, coletadas de forma aleatória em cada parcela, na camada de 0 a $20 \mathrm{~cm}$. As amostras foram analisadas de acordo com os procedimentos da EMBRAPA (1997). As seguintes variáveis químicas e físicas do solo de cada parcela foram determinadas: $\mathrm{pH}$, níveis de fósforo $(\mathrm{P})$, potássio $(\mathrm{K})$, cálcio $(\mathrm{Ca})$, magnésio $(\mathrm{Mg})$, sódio $(\mathrm{Na})$, alumínio (Al) e matéria orgânica (M.O.), índices $\mathrm{SMP}$, acidez potencial $(\mathrm{H}+\mathrm{Al})$, capacidade de troca

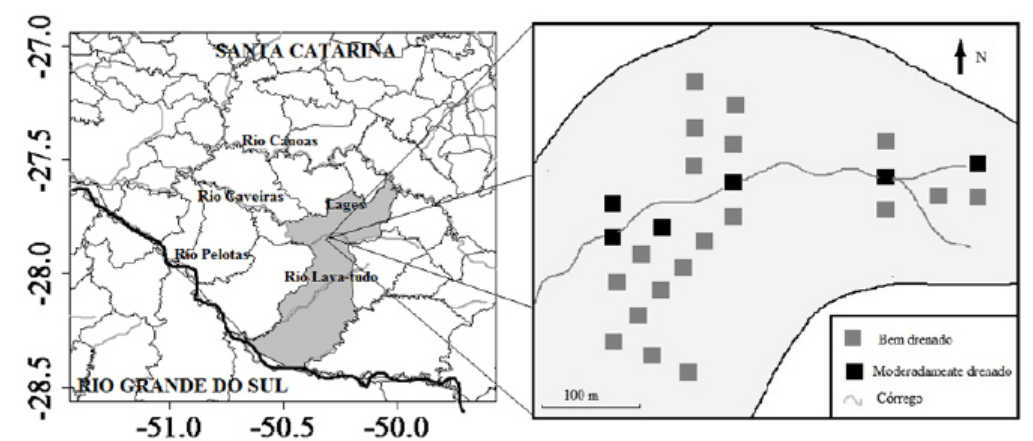

FIGURA 1: Localização da área de estudo e distribuição das parcelas no fragmento de Floresta com Araucária no município de Lages, SC.

FIGURE 1: Location of the study area and plots distribution in an Araucaria Forest fragment, in the municipality of Lages, SC state. 
catiônica efetiva $(\mathrm{t})$, saturação de $\mathrm{Al}$ na capacidade troca catiônica $(\mathrm{m})$, soma de bases (SM), saturação por base na CTC efetiva (V) e os teores de areia, silte e areia. A resistência do solo à penetração foi avaliada em nove pontos distribuídos de forma sistemática em cada parcela, dos quais foi retirada uma média por parcela, até $20 \mathrm{~cm}$ de profundidade do solo, por meio de um penetrômetro digital modelo penetrolog PLG1020 Falker, utilizando o cone tipo 2, com leituras em condição de umidade do solo correspondente à capacidade de campo. A profundidade efetiva do solo foi considerada como a soma dos horizontes A e B do solo (SANTOS et al., 2005), determinada em nove pontos em cada parcela.

A cobertura do dossel foi medida por meio de leituras com densiômetro esférico (Modelo A) (LEMMON, 1956), realizadas em novembrodezembro, a partir de quatro leituras no centro de cada parcela.

\section{Análise dos dados}

Após a verificação exploratória da distribuição dos valores das variáveis analisadas e de suas respectivas variâncias, bem como da natureza desbalanceada em relação às classes de drenagem, de acordo com as recomendações de Logan (2011), optou-se pela comparação das variáveis ambientais e estruturais (densidade e área basal) entre os setores por meio do teste não paramétrico de Mann-Whitney. A comparação do número de espécies foi realizada utilizando-se o procedimento de rarefação, considerando para o cálculo a quantidade de indivíduos do local de menor densidade (300 indivíduos), e da organização da comunidade, por meio da análise de variância multivariada não paramétrica (NPMANOVA).

Os dados de abundância das espécies em cada uma das parcelas foram ordenados por meio da técnica multivariada NMDS. Em seguida, as variáveis ambientais foram ajustadas à ordenação produzida pela NMDS e, aquelas significativas $(\mathrm{p}<0,05)$, plotadas a posteriori na forma de vetor. Foram determinadas as espécies de maior associação a cada classe de drenagem por meio da análise de espécies indicadoras (DUFRÊNE e LEGENDRE, 1997).

Toda a análise dos dados foi feita no programa estatístico R (R DEVELOPMENT CORE TEAM, 2011), com a utilização do pacote Vegan (OKSANEN et al., 2012) para a rarefação, NMDS e
MPMANOVA, e Labdsv (ROBERTS, 2012) para a definição das espécies indicadoras.

\section{RESULTADOS}

As parcelas contemplaram duas classes de drenagem representando locais bem drenados, onde a água se infiltra com facilidade, predominantemente nas encostas, e locais moderadamente drenados, onde a água se infiltra lentamente, com o solo predominantemente úmido, próximos do curso de água (Figura 1). Estes dois setores de drenagem

TABELA 1: Variáveis ambientais amostradas em dois setores (BD: bem drenado; MD: moderadamente drenado) em um Fragmento de Floresta Ombrófila Mista em Lages, SC.

TABLE 1: Environmental variables sampled in two sectors (BD: well-drained; MD: moderately-drained) in an Araucaria Forest fragment in the municipality of Lages, SC state.

\begin{tabular}{|c|c|c|c|}
\hline & $\mathrm{BD}$ & MD & $p$ \\
\hline Número de parcelas & 16 & 06 & \\
\hline $\mathrm{pH}$ & 4,78 & 4,95 & 0,3145 \\
\hline $\mathrm{Ca}^{2+}\left(\mathrm{cmol}_{\mathrm{c}} \mathrm{dm}^{-3}\right)$ & 3,74 & 5,11 & 0,2736 \\
\hline $\mathrm{Mg}^{2+}\left(\mathrm{cmol}_{\mathrm{c}} \mathrm{dm}^{-3}\right)$ & 0,59 & 0,85 & 0,1346 \\
\hline $\mathrm{Al}^{1+}\left(\mathrm{cmol}_{\mathrm{c}} \mathrm{dm}^{-3}\right)$ & 1,53 & 0,69 & $0,0857^{1}$ \\
\hline $\mathrm{H}+\mathrm{Al}\left(\mathrm{cmolc} / \mathrm{dm}^{3}\right)$ & 15,3 & 11,9 & 0,1591 \\
\hline CTC efetiva $\left(\mathrm{cmolc} / \mathrm{dm}^{3}\right)$ & 6,03 & 6,83 & 0,6791 \\
\hline Saturação por base (\%) & 23,56 & 34,93 & 0,2465 \\
\hline M.O. (\%) & 5,46 & 4,35 & $0,0689^{1}$ \\
\hline $\mathrm{P}(\mathrm{mg} / \mathrm{dm})$ & 3,36 & 3,40 & 0,6772 \\
\hline $\mathrm{Na}(\mathrm{mg} / \mathrm{dm})$ & 4,37 & 4,33 & 0,6949 \\
\hline $\mathrm{K}(\mathrm{mg} / \mathrm{dm})$ & 65,95 & 72,33 & 0,3896 \\
\hline CTC a $\mathrm{pH}=7\left(\mathrm{cmolc} / \mathrm{dm}^{3}\right)$ & 19,79 & 18,05 & 0,4640 \\
\hline Areia (\%) & 50,21 & 45,00 & 0,2782 \\
\hline Silte $(\%)$ & 30,16 & 36,50 & $0,0382^{*}$ \\
\hline Argila (\%) & 19,53 & 18,50 & 0,8482 \\
\hline Cobertura do dossel (\%) & 87,62 & 89,51 & 0,2641 \\
\hline Resistência à penetração (Kpa) & 965,90 & 856,62 & 0,2212 \\
\hline Cota média (m) & 992,30 & 978,40 & $0,0485^{*}$ \\
\hline Desnível máximo (m) & 9,57 & 9,12 & 0,9746 \\
\hline Declividade média $\left({ }^{\circ}\right)$ & 15,29 & 13,40 & 0,3895 \\
\hline Profundidade do solo $(\mathrm{cm})$ & 28,35 & 36,58 & 0,6419 \\
\hline
\end{tabular}

Em que: $p=$ Significância, ${ }^{1}$ Significativo a $10 \%$ de probabilidade de acordo com o teste Mann-Whitney; *significativo a $5 \%$ de probabilidade de acordo com o teste Mann-Whitney. 
apresentaram diferenças em relação às características ambientais analisadas (Tabela 1).

As parcelas classificadas como bem drenadas (BD) apresentaram maiores valores de cota média, de teores de $\mathrm{Al}$ e concentração de matéria orgânica. Já os locais moderadamente drenados (MD) apresentaram solos mais siltosos. Ambas as classes de drenagem apresentaram dois tipos de solos, Cambissolos Húmicos (BD: 10 parcelas; MD: quatro parcelas) e Neossolos Litólicos (BD: nove parcelas; MD: duas parcelas).

Apesar das diferenças ambientais, os valores médios de número de indivíduos e de área basal não diferiram entre os setores (Tabela 2). No entanto, o número de espécies do setor "Moderadamente Drenado" apresentou menor número de espécies.

$\mathrm{Na}$ ordenação produzida pela NMDS (stress $=0,1761)$, é possível verificar que os setores apresentaram uma partição florístico-estrutural (Figura 2), que foi confirmado pelo NPMANOVA $(p<0,005)$. Sete variáveis $(\mathrm{pH}$, saturação por base, $\mathrm{Mg}$, compactação do solo, $\mathrm{H}+\mathrm{Al}, \mathrm{Al}$ e declividade média) apresentaram relação significativa $(p \leq 0,05)$ com a ordenação produzida. A variação ao longo do gradiente associada, principalmente, à saturação por base (SatBa) e teores de Al, entre outras variáveis associadas, esteve relacionada à dicotomitização produzida pelas classes de drenagem. Já declividade média (DecMed) explicou a variação da comunidade dentro de cada setor.

O setor "bem drenado" apresentou três espécies indicadoras: Podocarpus lambertii Klotzsch ex Endl., Myrcia splendens (Sw.) DC. e
Zanthoxylum kleinii (R.S.Cowan) P.G.Waterman. No setor "moderadamente drenado", as espécies indicadoras foram Banara tomentosa Clos, Campomanesia xanthocarpa O.Berg, Xylosma ciliatifolia (Clos) Eichler, Nectandra megapotamica (Spreng.) Mez, Oreopanax fulvus Marchal e Solanum pabstii L.B.Sm. \& Downs (Tabela 3).

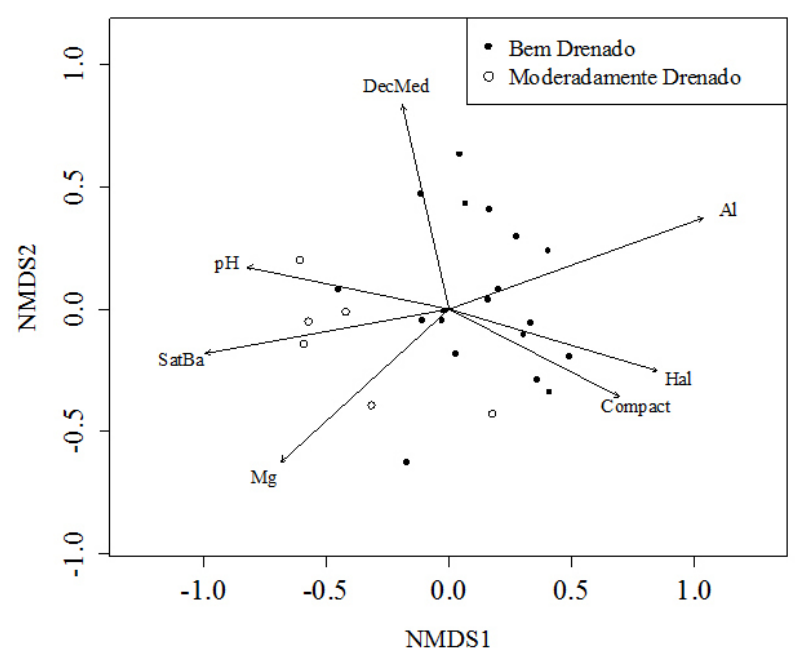

FIGURA 2: Ordenação das parcelas por meio da NMDS e vetores das variáveis ambientais significativas $(p \leq 0,05)$ em um fragmento de Floresta Ombrófila Mista, em Lages, SC.

FIGURE 2: Plot ordination produced by a NMDS and significative $(\mathrm{p} \leq 0,05)$ environmental variables vectors in an Araucaria Forest fragment in the muncipality of Lages, SC state.

TABELA 2: Valores médios de número de indivíduos e de área basal e riqueza determinada a partir da rarefação, com respectivos valores de erro padrão nos setores (BD: bem drenado; MD: moderadamente drenado) avaliados em um fragmento de Floresta Ombrófila Mista, em Lages, SC.

TABLE 2: Tree individuals and basal area mean values and richness determined by rarefaction, with respective standards errors by sectors (BD: well-drained; MD: moderately-drained) evaluated in an Araucaria Forest fragment, in the municipality of Lages, SC state.

\begin{tabular}{lccc}
\hline & $\mathrm{BD}$ & $\mathrm{MD}$ & $p$ \\
\hline Número médio de indivíduos & $73,65 \pm 5,88$ & $62,00 \pm 6,49$ & $0,2090 \mathrm{Ns}$ \\
Área basal média $\left(\mathrm{m}^{2}\right)$ & $1,45 \pm 0,10$ & $1,29 \pm 0,25$ & $0,3706 \mathrm{Ns}$ \\
Número de espécies & $56,76 \pm 3,05$ & $49,57 \pm 0,63$ & 1
\end{tabular}

Em que: $p=$ Significância, Ns = não significativo de acordo com o teste Mann-Whitney. ${ }^{1}$ Valores diferentes de acordo com o método de rarefação com 300 indivíduos. 
TABELA 3: Espécies Indicadoras dos setores "bem drenado" (BD) e "moderadamente drenado" (MD) em um fragmento de Floresta com Araucária, em Lages, SC.

TABLE 3: Indicator species for the sectors well-drained (BD) and moderately-drained (MD) in an Araucaria Forest fragment in the muncipality of Lages, SC state.

\begin{tabular}{lccc}
\hline \multicolumn{1}{c}{ Espécies Indicadoras } & Setor & Valor de indicação & $p$ \\
\hline Podocarpus lambertii Klotzsch ex Endl. & $\mathrm{BD}$ & 0,7083 & 0,014 \\
Myrcia splendens (Sw.) DC. & $\mathrm{BD}$ & 0,6995 & 0,049 \\
Zanthoxylum kleinii (R.S.Cowan) P.G.Waterman & $\mathrm{BD}$ & 0,6714 & 0,045 \\
Banara tomentosa Clos & $\mathrm{MD}$ & 0,7671 & 0,006 \\
Campomanesia xanthocarpa (Mart.) O.Berg & $\mathrm{MD}$ & 0,7395 & 0,006 \\
Xylosma ciliatifolia (Clos) Eichler & $\mathrm{MD}$ & 0,5872 & 0,018 \\
Nectandra megapotamica (Spreng.) Mez & $\mathrm{MD}$ & 0,5830 & 0,027 \\
Oreopanax fulvus Marchal & $\mathrm{MD}$ & 0,5390 & 0,030 \\
Solanum pabstii L.B.Sm. \& Downs & $\mathrm{MD}$ & 0,5067 & 0,020 \\
\hline
\end{tabular}

\section{DISCUSSÃO}

A posição topográfica pode determinar a capacidade de drenagem e as propriedades físicas e químicas do solo (SOLLINS, 1998). Como o fragmento florestal estudado está localizado numa pequena bacia hidrográfica, este padrão se repetiu, com parcelas de menor cota média, próximas do curso da água, apresentando drenagem moderada, em contraste com as parcelas com maior distância vertical do curso da água, que apresentaram boa drenagem.

Também estiveram relacionados às parcelas bem drenadas os maiores teores de $\mathrm{Al}$ e matéria orgânica(M.O.), estasúltimas variáveis normalmente associadas entre si (EBELING et al., 2008). No entanto, não eram esperados menores teores de M.O. nos locais mais úmidos, pois, em ambientes menos drenados, normalmente, há o seu acúmulo. Porém, esse não é o caso do presente estudo que, apesar de moderadamente drenado, provavelmente não caracteriza um ambiente hipóxido ou anóxido, o qual iria determinar o acúmulo de M.O. Dessa forma, a menor concentração de M.O. no ambiente mais úmido (moderadamente drenado) pode ser o resultado do favorecimento da decomposição da M.O. nestes ambientes, como observado por Van den Berg e Oliveira-Filho (1999). Nesse ambiente mais úmido também foi observada diferença física relacionada à maior concentração de silte, padrão esperado, já que siltes são caracterizados como partículas leves que tendem a se acumular em cotas mais baixas, geralmente definindo um solo com menor drenagem.

A heterogeneidade ambiental, representada predominantemente pela drenagem do solo, apesar de não influenciar o número de indivíduos e a área basal, teve influência significativa sobre a riqueza e a organização florística e estrutural do componente arbóreo. $\mathrm{O}$ menor número de espécie no ambiente como menor capacidade de infiltração de água no perfil do solo reforça o padrão encontrado em ecossistemas florestais em vários outros trabalhos (GAMA et al., 2005; CURCIO et al., 2006; SILVA et al., 2007; SILVA et al., 2010; PRATA et al., 2011), confirmando que a saturação hídrica do solo representa um fator seletivo para ocorrência de espécies (KOZLOWSKI, 2002), diminuindo, assim, a riqueza observada nestas áreas.

Como consequência da seletividade, foi possível observar a partição da organização espacial da comunidade em função das classes de drenagem existentes. Enquanto características edáficas, predominantemente teor de $\mathrm{Al}$ e soma de bases, estiveram associadas com a variação da comunidade relacionada às classes de drenagem, a declividade média das parcelas esteve associada com a variação da comunidade dentro de cada classe. Este resultado demonstra a natureza multivariada de ecossistemas florestais naturais, como frequentemente relatado na literatura (e.g. OLIVEIRA-FILHO et al., 2001; PEREIRA et al., 2007) e ilustra que a distribuição espacial das espécies arbóreas é o resultado de complexa interação entre diferentes variáveis, que define o nicho ecológico efetivo das espécies.

A ocorrência de três espécies indicadoras no 
setor bem drenado, Podocarpus lambertii, Myrcia splendens e Zanthoxylum kleinii, e seis espécies no setor com menor capacidade de drenagem, com destaque para Banara tomentosa, Campomanesia xanthocarpa e Nectandra megapotamica, é o resultado da partição espacial da comunidade. Algumas espécies listadas como indicadoras de um setor específico já foram relatadas como preferências das mesmas condições em outros trabalhos, como Myrcia splendens, em solos bem drenados, (TEIXEIRA e ASSIS, 2009) e Campomanesia xanthocarpa e Nectandra megapotamica em ambientes com saturação hídrica do solo (BOTREL et al., 2002; CARVALHO et al., 2005). No entanto, devido à complexa interação entre os fatores que determinam a ocorrência de uma espécie, como já discutido anteriormente, os resultados encontrados não podem ser generalizados, uma vez que uma espécie classificada como indicadora no presente estudo, em outras circunstâncias, pode apresentar outras associações com o ambiente. Por exemplo, Curcio et al. (2006) observaram Podocarpus lambertii, classificado como indicador do local bem drenado no presente estudo, ocorrendo em diferentes condições em relação à infiltração da água no solo, sugerindo que esta espécie pode apresentar elevada plasticidade no que se refere a esta característica ambiental.

\section{CONCLUSÃO}

No fragmento de Floresta com Araucária estudado, as variações ambientais associadas à drenagem do solo representaram um fator determinante na heterogeneidade da comunidade de espécies arbóreas. Esta observação tem grande implicação na definição de estratégias de conservação e restauração desse tipo de ecossistema, pois ilustra a importância do uso de espécies adequadas de acordo com o ambiente considerado.

\section{AGRADECIMENTO}

Ao CNPq, pelo auxílio financeiro por meio do Edital Universal, processo 475847/2009-1.

\section{REFERÊNCIAS BIBLIOGRÁFICAS}

BARDDAL, M. L. et al. Caracterização florística e fitossociológica de um trecho sazonalmente inundável de floresta aluvial, em Araucária, PR. Ciência Florestal, Santa Maria, v. 14, n. 2, p. 35-70, jul-dez, 2004.

BOTREL, R. T. et al. Influência do solo e topografia sobre as variações da composição florística e estrutura da comunidade arbóreo-arbustiva de uma floresta estacional semidecidual em Ingaí, MG. Revista Brasileira de Botânica, São Paulo, v. 25, n. 2, p. 195-213, jun. 2002.

BUDKE, J. C. et al. Tree community features of two stands of riverine forest under different flooding regimes in Southern Brazil. Flora, Amsterdam, v. 203, n. 2, p. 162-174, Aug./Sept.. 2008.

CARVALHO, D. A. et al. Distribuição de espécies arbóreo-arbustivas ao longo de um gradiente de solos e topografia em um trecho de floresta ripária do Rio São Francisco em Três Marias, MG, Brasil.

Revista Brasileira de Botânica, São Paulo, v. 28, n. 2, p. 329-345, abr./jun. 2005.

CARVALHO, J. et al. Relações entre a distribuição das espécies de diferentes estratos e as características do solo de uma floresta aluvial no Estado do Paraná, Brasil. Acta Botanica Brasilica, São Paulo, v. 23, n.1, p. 1-9, jan/mar, 2009.

CHASE, M. W. et al. An update of the Angiosperm Phylogeny Group classification for the orders and families of flowering plants: APG III. Botanical Journal of the Linnean Society, Londres, v. 161, n. 2, p. 105-121, Oct. 2009.

CURCIO, G. R. et al. Compartimentação topossequencial e caracterização fitossociológica de um Capão de Floresta Ombrófila Mista. Floresta, Curitiba, v. 36, n. 3, p. 361-369, set./dez. 2006.

DUFRÊNE, M.; LEGENDRE, P. Species assemblages and indicator species: the need for a flexible asymmetrical approach. Ecological monographs, Ithaca, v. 67 , n. 3, p. 345-366, Aug. 1997.

EBELING, A. G. et al. Relação entre acidez e outros atributos químicos em solos com teores elevados de matéria orgânica. Bragantia, Campinas, v. 67, n. 2, p. 429-439, abr./jun. 2008.

EMBRAPA. Manual de métodos de análise de solo. Rio de Janeiro: Serviço Nacional de Levantamento e Conservação de Solo. 1997. 212 p. EMBRAPA. Sistema brasileiro de classificação de solos. Rio de Janeiro: EMBRAPA Solos. 2006. $306 \mathrm{p}$.

EMBRAPA. Solos do Estado de Santa Catarina. Rio de Janeiro: EMBRAPA Solos. 2004. 726 p. (Boletim de Pesquisa e Desenvolvimento, 46). GAMA, J. R. V. et al. Comparação entre florestas de várzea e de terra firme do Estado do Pará. Revista Árvore, Viçosa, v. 29, n. 4, p. 607-616, jul./ago. 
2005

GOMES, M. A. F. et al. Caracterização pedomorfoclimática das áreas de afloramento do Aquífero Guarani no Brasil: Base para uma proposta de gestão sustentável. In: GOMES, M. A. F. Uso agrícola das áreas de afloramento do Aquífero Guarani no Brasil: Implicações para a água subterrânea e propostas de gestão com enfoque agroambiental. Brasília: Embrapa Informação Tecnológica, 2008. p. 47-64.

HIGUCHI, P. et al. Floristic composition and phytogeography of the tree component of Araucaria Forest fragments in southern Brazil. Brazilian Journal of Botany, São Paulo, v. 35, n. 2, p. 145-157, abr./jun. 2012a.

HIGUCHI, P. et al. Influência de variáveis ambientais sobre o padrão estrutural e florístico do componente arbóreo, em um fragmento de Floresta Ombrófila Mista Montana em Lages, SC. Ciência Florestal, Santa Maria, v. 22, n. 1, p. 79-90, jan./mar. 2012b.

IBGE. Manual técnico da vegetação brasileira. Rio de Janeiro: Fundação Instituto Brasileiro de Geografia e Estatística, 1992. 92 p. (Série: Manuais técnicos em geociências; n.1).

KOZLOWSKI, T. T. Physiological-ecological impacts of flooding on riparian forest ecosystems. Wetlands, Whashington, v. 22, n. 3, p. 550-561, Sept. 2002.

LEMMON, P. A spherical densiometer for estimating forest overstory density. Forest Science, Bethesda, v. 2, n.1, p. 314-320, June 1956.

LOGAN, M. Biostatistical design and analysis using R: a practical guide. [S.1.]: Wiley-Blackwell. 2011. $546 \mathrm{p}$.

OKSANEN, J. et al. Vegan: Community Ecology Package. Disponível em: < (http://cran.r-project.org/ package $=$ vegan $)>$ Acesso em: 30 de julho de 2012 OLIVEIRA FILHO, A. T. et al. Differentiation of streamside and upland vegetation in an area of montane semideciduous forest in southeastern Brazil. Flora, Amsterdam, v. 189, n. 4, p. 287-305, Dec. 1994.

OLIVEIRA FILHO, A. T. et al. Tree species distribution along soil catenas in a riverside semideciduous forest in southeastern Brazil. Flora, Amsterdam, v. 192, n. 1, p. 47-64, Jan. 1997.

OLIVEIRA FILHO, A. T. et al. Variation in tree community composition and structure with changes in soil properties within a fragment of semideciduous forest in south-eastern Brazil. Edinburgh Journal of Botany, Cambridge, v. 58, n. 1, p. 139-158,
Jan./Mar. 2001

PEREIRA, J. A. A.; OLIVEIRA FILHO, A. T.; LEMOS FILHO, J. P. Environmental heterogeneity and disturbance by humans control much of the tree species diversity of Atlantic montane forest fragments in SE Brazil. Biodiversity and Conservation, London, v. 16, n. 6, p. 1761-1784, Oct. 2007.

PIMENTA, J. A. et al. Adaptations to flooding by tropical trees: morphological and anatomical modifications. In: SCARANO, F. R.; FRANCO, A. C. Ecophysiological strategies of xerophytic and amphibious plants in the neotropics. Oecologia Brasiliensis Series, Rio de Janeiro, v. 4, n. 1, p. 157-176, jan. 1998.

PRATA, E. M. B. et al.. Fitossociologia e distribuição de espécies arbóreas em uma floresta ribeirinha secundária no município de Rio Claro, SP, Brasil. Revista Brasileira de Botânica, São Paulo, v. 34, n. 2, p. 159-168, abr./jun. 2011.

R DEVELOPMENT CORE TEAM. R: A language and environment for statistical computing. R Foundation for Statistical Computing, 2008. Disponível em: $<$ (http://www.r-project.org) $>$ Acesso em: 18 de novembro de 2011.

RIBEIRO, M. C. et al. The Brazilian Atlantic Forest: How much is left, and how is the remaining forest distributed? Implications for conservation. Biological Conservation, Amsterdam, v. 142, n. 6, p. 1141-1153, June 2009.

ROBERTS, D. W. Labdsv: Ordination and multivariate analysis for ecology. Disponível em: $\quad<$ (http://cran.r-project.org/package=labdsv) $>$ Acesso em 30 de julho de 2012.

SANTOS, R. D. et al. Manual de descrição e coleta de solo no campo. 5. ed. Viçosa: SBCS. 2005. 92 p. SILVA, A. C. et al. Comparação florística de florestas inundáveis das regiões Sudeste e Sul do Brasil. Revista Brasileira de Botânica, São Paulo, v. 30, n. 2, p. 257-269, abr.jun. 2007.

SILVA, A. C. et al. Florestas Inundáveis: ecologia, florística e adaptações das espécies. Lavras: Ed. da UFLA, 2012a. $168 \mathrm{p}$.

SILVA, A. C. et al. Florística e estrutura da comunidade arbórea em fragmentos de floresta aluvial em São Sebastião da Bela Vista, Minas Gerais, Brasil. Revista Brasileira de Botânica, São Paulo, v. 32, n. 2, p. 283-297, abr./jun. 2009.

SILVA, A. C. et al. Relações florísticas e fitossociologia de uma Floresta Ombrófila Mista Montana secundária em Lages, Santa Catarina. Ciência Florestal, Santa Maria, v. 22, n. 1, 
p. 193-206, jan. 2012b.

SILVA, A. C.; HIGUCHI, P.; VAN DEN BERG, E. Effects of soil water table regime on tree community species richness and structure of alluvial forest fragments in Southeast Brazil. Brazilian Journal of Biology, São Carlos, v. 70, n. 3, p. 465-471, Aug. 2010 .

SOLLINS, P. Factors influencing species composition in tropical lowland rain forest: does soil matter? Ecology, Ithaca, v. 79, n. 1, p. 23-30, Jan. 1998.

STEEGE, H. T. et al. Tropical rain forest types and soil factors in a watershed area in Guyana. Journal of Vegetation Science, Hoboken, v. 4, n. 5, p. 705-716, Sept. 1993.
TEIXEIRA, A. P.; ASSIS, M. A. Relação entre heterogeneidade ambiental e distribuição de espécies em uma floresta paludosa no Município de Cristais Paulista, SP, Brasil. Acta Botânica Brasilica, São Paulo, v. 23, n. 3, p. 843-853, July/ Sept. 2009.

VAN DEN BERG, E.; OLIVEIRA-FILHO, A. T. Spatial partitioning among tree species within an area of tropical montane gallery forest in south-eastern Brazil. Flora, Amsterdam, v. 194, n. 3, p. 249-266, July 1999. WEBB, C. O. et al. Phylogenies and Community Ecology. Annual Review of Ecology and Systematics, New York, v. 33, p. 475-505, Aug. 2002 . 